\title{
Eltern und Smartphones
}

\section{Auswirkungen auf die Kinder}

\author{
M. Spitzer, Ulm
}

Man kann es täglich und überall beobachten: Die lieben Kleinen backen Sandkuchen, klettern auf Bäume, essen oder sitzen einfach nur im Kinderwagen - und Mama oder Papa schauen auf ihr Smartphone. Eltern und Kinder sind zwar räumlich nahe beisammen, geistig jedoch nicht miteinander. "So ist das eben heute", mag der Leser etwas frustriert kommentieren, „da kann man nichts machen, die Zeiten ändern sich." Die Frustration rührt daher, dass man sich bei solchen Beobachtungen des Eindrucks nicht erwehren kann, dass hier etwas schiefläuft. Die Eltern sind abwesend und die Kinder wollen deren Aufmerksamkeit. Das nervt die Eltern und sie wenden sich erst recht ab. Die Kinder quengeln noch heftiger und die Sache eskaliert nicht selten. Oder die Kinder wenden sich auch $\mathrm{ab}$ und es geschieht - nichts.

Was bedeutet das für die Familie? Hat das Auswirkungen, und wenn ja, welche? Wie häufig kommt das überhaupt vor? Ist das bei Vater und Mutter gleich? Und was bewirkt das langfristig bei den Kindern?

Was im öffentlichen Raum wie beispielsweise auf vielen Spielplätzen und auch daheim mittlerweile den ganz normalen Alltag darstellt, wird seit wenigen Jahren wissenschaftlich untersucht. Das ist wichtig, denn man könnte die eben erwähnte Frustration von Nutzern und vor allem von Menschen in deren Umgebung ja einfach den fortschrittsfeindlichen älteren Beobachtern anlasten, den ewig Gestrigen, für die früher eben immer alles besser war. Wenn die Beobachtungen jedoch zutreffen und sie zum Alltag vieler Menschen und vor allem Kindern gehören, sollten wir uns darüber Gedanken machen.

Nervenheilkunde 2018; 37: 469-477

Korrespondenzadresse

Prof. Dr. Dr. Manfred Spitzer, Universitätsklinikum Ulm Klinik für Psychiatrie und Psychotherapie III

Leimgrubenweg 12, 89075 Ulm
Kinder machen nach, was ihre

Eltern tun, auch und gerade im

Hinblick auf den Medienkonsum.

Kinder machen nach, was ihre Eltern tun, auch und gerade im Hinblick auf den Medienkonsum. Das Medienverhalten von Eltern überträgt sich auf die Kinder, wie man schon seit geraumer Zeit weiß: Schauen die Eltern viel fern, tun es die Kinder auch. Dies trifft für die mittlerweile hinzugekommenen digitalen Medien - Computer, Tablet, Video, Spiele-Konsole, Smartphone auch zu. Ein lustiges Foto oder Video, die neueste Nachricht von Opa oder der Tante - man schaut gemeinsam auf das Smartphone. Nach einer repräsentativen Studie aus den USA an 1786 Eltern von Kindern im Alter von 8 bis 18 Jahren verbringen diese täglich im Mittel 9 Stunden und 22 Minuten mit Medien, davon eine Stunde und 39 Minuten bei der Arbeit und 7 Stunden 43 Minuten in der Freizeit $(\triangleright$ Tab. 1).

Die Daten der Studie zeigen zudem: 51\% der Eltern geben an, mehr als 8 Stunden täglich mit Medien zu verbringen, nur $19 \%$ der Eltern geben dagegen weniger als 4 Stunden tägliche Medienzeit an. Fast alle Eltern geben an, dass sie "gestern" Medien genutzt haben, $d$. h. fast niemand (1\%) verbringt gelegentlich „einen Tag ohne Medien“. Das passive Betrachten (Fernsehen, DVDs oder Videos schauen) ist dabei mit 91\% die häufigste Beschäftigung mit Medien („was haben Sie gestern gemacht?"), am wenigsten hingegen werden E-Book Reader (19\%) verwendet (12, S. 7).

Trotz dieser extremen Mediennutzung (9:22 Stunden; d. h. mehr als die Hälfte der im Wachen verbrachten Zeit!) geben 78\% der Eltern ( $81 \%$ der Mütter und $74 \%$ der Väter) an, dass sie im Hinblick auf die Mediennutzung ein gutes Vorbild für ihre Kinder seien. Zugleich sagen 37\% der Eltern, dass das Aushandeln der täglichen Mediennutzungszeit ihrer Kinder Konflikte verursacht, und nur 35\% gaben an, dass die neuen Technologien - Smartphones und Tablets - das Leben von Eltern einfacher machen würden. In einer anderen USamerikanischen Studie an 2326 Eltern von Kindern im Alter bis 8 Jahren gaben ebenfalls nur 29\% an, dass Smartphone und Tablet ihnen das Eltern-Sein einfacher machen (34, S. 4). Aus diesen Daten kann man folgern:

- Eltern sind sich über das Ausmaß ihrer Mediennutzung nicht im Klaren,

- über ihre diesbezügliche Vorbildfunktion für ihre Kinder auch nicht und

- sie leiden darunter.

- Tabelle 1 zeigt weiterhin: Je gebildeter die Eltern sind, desto weniger Zeit verbringen sie mit Medien. Je mehr sie verdienen, desto weniger Freizeit und desto mehr Arbeitszeit verbringen sie jeweils mit digitaler Informationstechnik. Diese Effekte sind linear, und die Unterschiede zwischen den einzelnen Gruppen sind jeweils signifikant.

Erfasst wurde in dieser Untersuchung (12) auch der Medienkonsum der Kinder der untersuchten Eltern. Der Besitz von Geräten ist bei Einzelkindern generell höher. Bei den Teenagern haben $80 \%$ einen eigenen Social Media Account, aber nur $40 \%$ der Eltern wissen gut darüber Bescheid, was ihre Kinder dort tun. Dem gegenüber meinen über $80 \%$ der Eltern, gut darüber Bescheid zu wissen, was die Kinder im Fernsehen oder Kino sehen. Eltern sorgen sich also über das gefährlichere Medium halb soviel. (Zur Gefährlichkeit von Facebook, vgl. 35, 36) Immerhin 56\% der Eltern sorgen sich um den suchterzeugenden Effekt von Bildschirmmedien, was mit dazu beitragen dürfte, dass $77 \%$ aller Eltern die Mediennutzung ihre Kinder in irgendeiner Weise reglementieren. 
Tab. 1 Mediennutzung im Hinblick auf Art und Inhalt (nach 12, S. 7). Social Media und Web-Browsing kann sowohl am Computer als auch am Smartphone oder Tablet erfolgen. Die ethnische Zugehörigkeit wurde durch Selbst-Identifikation ${ }^{1}$ bestimmt.

\begin{tabular}{|l|l|l|l|l|l|l|l|l|l|l|l|l}
\hline & & \multicolumn{3}{l}{ Bildungsgrad } & \multicolumn{3}{l}{ Ethnische Zugehörigkeit } & \multicolumn{2}{l}{ Einkommen } \\
\hline Was & Alle & niedrig & mittel & hoch & White & Black & Hispanic & niedrig & mittel & hoch \\
\hline Betrachten (TV, DVD, Video) & $3: 17$ & $4: 05$ & $3: 13$ & $2: 24$ & $2: 48$ & $4: 35$ & $4: 00$ & $4: 15$ & $3: 14$ & $2: 42$ \\
\hline Spiele (PC, Video, Konsole, Smartphone, Tablet) & $1: 30$ & $1: 56$ & $1: 31$ & $1: 00$ & $1: 11$ & $2: 29$ & $1: 56$ & $1: 53$ & $1: 36$ & $1: 04$ \\
\hline Social Media & $1: 06$ & $1: 15$ & $1: 01$ & $1: 00$ & $1: 00$ & $1: 12$ & $1: 06$ & $1: 15$ & $1: 06$ & $1: 00$ \\
\hline Web-Browsing & $0: 51$ & $0: 47$ & $0: 55$ & $0: 52$ & $0: 46$ & $1: 02$ & $0: 47$ & $0: 46$ & $0: 49$ & $0: 56$ \\
\hline E-Reader & $0: 15$ & $0: 16$ & $0: 14$ & $0: 14$ & $0: 12$ & $0: 23$ & $0: 17$ & $0: 16$ & $0: 14$ & $0: 14$ \\
\hline Anderes (am PC, Tablet, Smartphone) & $0: 44$ & $0: 45$ & $0: 48$ & $0: 40$ & $0: 41$ & $0: 56$ & $0: 46$ & $0: 50$ & $0: 42$ & $0: 45$ \\
\hline Freizeit & $7: 43$ & $9: 03$ & $7: 41$ & $6: 10$ & $6: 38$ & $10: 37$ & $8: 52$ & $9: 15$ & $7: 42$ & $6: 41$ \\
\hline Arbeit & $1: 39$ & $1: 06$ & $1: 57$ & $2: 03$ & $1: 44$ & $2: 05$ & $1: 09$ & $1: 05$ & $1: 31$ & $2: 13$ \\
\hline Gesamt & $9: 22$ & $10: 10$ & $9: 38$ & $8: 13$ & $8: 22$ & $12: 42$ & 10.01 & $10: 21$ & $9: 13$ & $8: 54$ \\
\hline
\end{tabular}

Etwa die Hälfte der Eltern glauben nicht, dass elektronische Medien ihre Kinder in positiver oder negativer Hinsichten beeinflussen.

Etwa die Hälfte der Eltern glauben nicht, dass elektronische Medien ihre Kinder in den verschiedensten Hinsichten (positiv oder negativ) beeinflussen und die andere Hälfte ist gespalten in Eltern, die vor allem positive Auswirkungen sehen und Eltern, die eher negative Auswirkungen bemerken. Über die vielfältigen Risiken und Nebenwirkungen von Smartphones für die Gesundheit und die Bildung ihrer Kinder sind $75 \%$ der Eltern also gar nicht informiert.

So nützlich solche Befragungen sind, um das Bild, das Eltern von sich selbst und ihren Kindern haben, zu ermitteln, so wichtig ist es auch, nachzusehen, was tatsächlich der Fall ist.

Hierzu wurden in den letzten 3 bis 5 Jahren Arbeiten publiziert, die den Auswirkungen des elterlichen Gebrauchs von Smartphones auf deren Kinder nachgingen. Bereits im Jahr 2013 erschien in den

1 „The term "black" refers to any respondents who self-identify as black, non-Hispanic. The term "white" refers to any respondents who self-identify as white, non-Hispanic. The term "Hispanic" refers to any respondents who self-identify as Hispanic," lautet hierzu die entsprechende Erläuterung im Text (12, S. 5).

2 Übersetzt etwa: „Der große Verbindungsabbruch: wie man die Kindheit und die Beziehungen in der Familie im digitalen Zeitalter schützt."
USA das Buch The big disconnect: Protecting childhood and family relationships in the digital age $e^{2}$, in dem eher anekdotisch (aber sehr eindrucksvoll) beschrieben wird, wie Kinder die Aufmerksamkeit und $\mathrm{Zu}$ wendung ihrer Eltern durch deren Nutzung elektronischer Medien verlieren und dadurch Schaden nehmen.

Ein Jahr danach wurde eine bemerkenswerte europäische Studie zu den Auswirkungen des Medienkonsums bei kleinen Kindern publiziert (8). Im Rahmen der IDEFICS (Identification and Prevention of Dietary and Lifestyle-Induced Health Effects in Children and Infants) Studie wurden in 8 europäischen Ländern (Belgien, Deutschland, Estland, Italien, Spanien, Schweden, Ungarn und Zypern) 3604 Kinder im Alter von 2 bis 6 Jahren im Hinblick auf ihren Medienkonsum (Fernsehen, Computer und elektronische Spiele) untersucht. In dieser prospektiven Kohortenstudie wurden die Kinder dann zwei Jahre später erneut untersucht, wofür die folgenden 6 Indikatoren von deren Wohlbefinden erfasst wurden: Probleme mit Gleichaltrigen (peers) und mit ihren Gefühlen, emotionales Wohlbefinden, Selbstvertrauen, familiäre Probleme und soziales Eingebundensein. Mit jeder Stunde mehr vor dem Fernseher oder Computer erhöhte sich die Wahrscheinlichkeit unerwünschter Auswirkungen auf die Outcome-Variablen „emotionale Probleme" und „familiäre Probleme“ auf das 1,2- bis 2-Fache.

Im Fachblatt Pediatrics wurde ebenfalls bereits im Jahr 2014 eine Beobachtungsstu- die publiziert, bei der man in insgesamt 15 Fast-Food-Restaurants in der Gegend von Boston Familien beim Mittag- oder Abendessen beobachtete (23). Bei 40 der insgesamt beobachteten 55 Familien-Mahlzeiten benutzten die betreuenden Erwachsenen - in der Regel Mutter, Vater oder beide Eltern - ihr Mobiltelefon. „Das dominierende Thema, das sich mit der Nutzung des Mobiltelefons während der Erwachsener-Kind-Interaktion als besonders eng verknüpft erwies, war das Ausmaß der Vertiefung des Betreuers in die Apparate ${ }^{\text {"3 }}$, schreiben die Autoren mit gewisser Verwunderung (25, S. e843). In 16 Fällen befassten sich die Erwachsenen nahezu während der gesamten Mahlzeit nur mit ihrem Smartphone und nicht mit ihren Kindern. Dabei telefonierten sie eher nicht, sondern tippten oder wischten auf ihrem Smartphone herum.

Mit den Worten der Autoren: „[...] viele Betreuer $(\mathrm{n}=16)$ verwendeten das Gerät fast andauernd während der Mahlzeit, aßen und redeten während sie auf ihr Gerät schauten, und legten es nur kurz ab, um etwas anderes zu tun. Dieses Muster des Gebrauchs war sowohl bei der Anwesenheit von einem oder von beiden Elternteilen, in allen Altersgruppen und bei Männern und Frauen gleichermaßen. Der höchste Grad der Absorption der Aufmerksamkeit durch

3 Im englischen Original: „The dominant theme salient to mobile device use and caregiver-child interaction was the degree of absorption in devices caregivers exhibited." 
das Gerät war nicht beim Telefonieren zu beobachten, sondern wenn auf dem Gerät mit dem Finger getippt oder gewischt wurde, weil dabei der Blick des Betreuers auf

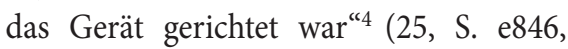
Übersetzung durch den Autor).

4 Originaltext: „[...] many caregivers $(\mathrm{n}=16)$ used the device almost continuously throughout the meal, eating and talking while looking at the device or only putting it down briefly to engage in other activities. This pattern of use occurred both with sole caregivers and those who had another adult present, of all age groups and both genders. The highest degree of absorption occurred when this nearly continuous use consisted of typing or making swiping finger motions, rather than with phone calls, because the caregiver's gaze was directed primarily at the device" $(25$, S. e846).
Das wiederum rief nicht selten den Protest der Kinder hervor, die begannen, laut zu werden und sich eigenartig zu verhalten, um die Aufmerksamkeit der Eltern auf sich $\mathrm{zu}$ ziehen. Dies wiederum führte $\mathrm{zu}$ vermehrtem Schimpfen oder kurzen genervten Strafaktionen seitens der Eltern, woraufhin die Situation nicht selten eskalierte. Dabei wurde beobachtet, wie die Eltern lauter wurden, aber weiter auf ihr Smartphone schauten und nicht selten eher roboterartig Ermahnungen wiederholten, ohne aufzumerken und das Kind anzusehen. Manchen Kindern war das Verhalten ihrer Eltern aber auch gleichgültig. $>\mathrm{Ta}$ belle 2 enthält Auszüge aus den schriftlich niedergelegten Beobachtungen von zuvor eigens in der anthropologischen Feldfor- schung und Beobachtung geschulten Personen. Die Lektüre stimmt nachdenklich, fragt man sich doch unwillkürlich, was wohl längerfristig aus den Kindern geworden sein mag.

Die eskalierende Spirale von

- Ablenkung der Eltern durch das Smartphone und

- den die Aufmerksamkeit ihrer Eltern beanspruchenden Kindern

beschreiben die Autoren sehr eindrücklich und heben hervor, dass es sich hier um ein immer wieder beobachtetes Verhaltensmuster der Familie handelte. Dabei hatte die ganze Unternehmung - eine Mahlzeit im Kreise der Familie - ja gerade ursprünglich den Zweck, dass die Familie

Tab. 2 Exzerpte aus der teilnehmenden Beobachtung von Familien beim Essen in Boston, USA (aus 25, Tabellen 2 und 3, gekürzt durch den Autor).

\begin{tabular}{|c|c|}
\hline Thema & Exzerpt \\
\hline Decreased responsiveness to child & $\begin{array}{l}\text { Female caregiver pulls out her phone from her purse and looks at it. Girl [school age] is talking to her caregi- } \\
\text { ver, caregiver is looking at the phone, nodding a little while the child talks but not looking back at her or res- } \\
\text { ponding with words. Caregiver doesn't appear to be listening but says a few words in response every once in a } \\
\text { while. Girl [...] keeps asking her questions but does not appear upset [...] with the few words that caregiver } \\
\text { says in response. Caregiver looks around the restaurant. Stares back at her phone. Child sways in her chair and } \\
\text { keeps eating fries and asking caregiver questions. Caregiver looks up occasionally to grab a French fry or } \\
\text { quickly say something to the girl and then continues to do something on her phone. }\end{array}$ \\
\hline $\begin{array}{l}\text { Decreased conversation with child; } \\
\text { child passive }\end{array}$ & $\begin{array}{l}\text { Female caregiver brings food over and sits down across from girl, they distribute the food and as [school aged] } \\
\text { girl starts eating caregiver brings out her smartphone. There is no conversation. Caregiver appears to be typing } \\
\text { into phone, holding it about } 10 \text { inches away from her face, looking into it for long stretches during which she } \\
\text { does not look up. She stops typing and is staring at the screen, touching it at points, holding it with her right } \\
\text { hand while she leans her chin on her left hand, her facial expression flat. She has been looking at it for about } 2 \\
\text { min without any change of gaze, while the girl eats and looks around the room. Caregiver then puts phone } \\
\text { down on the table and takes a drink from her smoothie. She then looks at the girl for about } 1-2 \text { s and then } \\
\text { down at her phone on the table. The girl keeps eating, then gets up to cross the room to get more ketchup. Ca- } \\
\text { regiver is not watching her do this; she is looking down at the phone. The girl quickly returns and sits and eats, } \\
\text { looking around the room while caregiver continues to hold the phone with her right hand and look at it, sip- } \\
\text { ping her drink without moving her gaze. She eats some fries slowly and continues to look at the phone with a } \\
\text { flat expression. Still no conversation. This continues through most of the meal. Now girl's head appears to be } \\
\text { looking right at caregiver, and caregiver looks up but not at the girl, scans the restaurant with a flat expression } \\
\text { and then eats some fries ... }\end{array}$ \\
\hline $\begin{array}{l}\text { Child escalation/ caregiver raising } \\
\text { voice during absorption }\end{array}$ & $\begin{array}{l}\text { Dad sits down with } 3 \text { boys and brings out a smartphone and starts swiping. Boys are talking to each other, ex- } \\
\text { cited, eating, talking, seeming to goof around. The dad looks up at them intermittently when they exclaim } \\
\text { something or raise their voices, but otherwise he looks at his phone, which he is holding in front of him. ...I can } \\
\text { see that dad is scrolling through small text; looks like a web site, not e-mail. Again dad looks up at them when } \\
\text { one of them exclaims something [inaudible] but then goes back to surfing web. [...] Oldest boy starts singing } \\
\text { "jingle bells, Batman smells," and the others try to join in but don't know the words. Dad not responding. } \\
\text { They're making up their own words to the song now. Dad calls the little one's name and speaks in a mildly } \\
\text { stern voice, and they stop singing. Little one laughs. Dad continues to hold phone up in front of face, looks } \\
\text { over to boys, then back to phone...The boys start singing "jingle bells, Batman smells" again, and dad looks } \\
\text { up and tells them to stop in a firm voice. Then he looks back to phone.... [end of meal] Again in stern voice, } \\
\text { seeming exasperated, dad says, "HURRY UP!" and the boys are being silly, licking each other's cones, climbing } \\
\text { on each other and on the divider between booths; dad keeps giving instructions in same tone of voice, says } \\
\text { something about not getting this again. }\end{array}$ \\
\hline
\end{tabular}


miteinander Zeit verbringt und kommuniziert. Dass Familienabendessen tatsächlich positive Auswirkungen auf die Kinder haben, wurde schon vor Jahren an dieser Stelle ausführlich anhand der dazu damals bereits vorhandenen wissenschaftlichen Literatur diskutiert (28). Das Smartphone torpediert jedoch offensichtlich den Sinn und Zweck gemeinsamer Mahlzeiten in Familien grundlegend. Es ist daher wohl auch kein Zufall, dass in der erwähnten Befragung (12, S. 24) nur 6\% aller Eltern angeben, ihren Kindern die Nutzung des Smartphones bei gemeinsamen Mahlzeiten zuhause $\mathrm{zu}$ erlauben. Das scheint für Schnellrestaurants nicht zu gelten: Nach einer kürzlich publizierten Beobachtungsstudie (mit ähnlichem ethnografischem Vorgehen wie gerade beschrieben) an $300 \mathrm{Fa}$ milien mit 450 Kindern im Alter von 2 bis 12 Jahren verwendeten $40 \%$ der Kinder und 70\% der Erwachsenen digitale Endgeräte (vor allem Smartphones, zuweilen auch Tablets oder Spielekonsolen) während der Mahlzeit (10).

Das Smartphone torpediert jedoch offensichtlich den Sinn und Zweck gemeinsamer Mahlzeiten in Familien grundlegend.

Nachdem die Arbeitsgruppe um die Kinderärztin Jenny Radesky mittels teilnehmender Beobachtung in Schnellrestaurants die Verhaltensauffälligkeiten von Eltern und Kindern erst einmal qualitativ beschrieben hatte, wurden auch Studien im Labor durchgeführt (23). Dort wurden Ton- und Filmaufnahmen gemacht, die danach unter Verwendung bestimmter Kriterien auch quantitative Auswertungen zuließen.

In den USA gibt es seit dem Jahr 1965 das Head Start Programm zur Bekämpfung

5 Das Programm wurde 1981 erweitert und 2007 nochmals autorisiert, hatte im Jahr 2005 insgesamt 22 Millionen Kinder eingeschlossen und im Jahr 2011 sieben Milliarden US\$ gekostet. Es gibt das Programm noch immer, sein Erfolg ist jedoch umstritten. Diese Fakten sind dem lesenswerten englischen Wikipedia-Artikel „Head Start (program)“ entnommen. (https://en.wikipedia.org/w/index. php?title=Head_Start_(program)\&oldid=847457185; abgerufen am 1.7.2018) der Folgen von Armut und zur Förderung von Kindern aus entsprechenden Famili$e^{5}$. Im Rahmen dieses Programms wurden zahlreiche Studien durchgeführt. Aus einer dieser Studien stammten Videoaufnahmen (aufgenommen zwischen Juni 2011 und Mai 2013) an 225 Müttern, die zusammen mit ihren sechsjährigen Kindern im psychologischen Labor eine Mahlzeit zu sich nahmen („structured eating task"). Man hatte diese Videoaufnahmen ursprünglich nur zum Zweck der Einschätzung der Mutter-Kind-Beziehung in einer ruhigen und störungsfreien Umgebung (und daher nicht „Zuhause“) erhoben. Dabei war aufgefallen, dass die Mütter nicht selten während der Mahlzeit ein mobiles digitales Endgerät (Smartphone, Tablet) verwendeten. Genau deswegen entschloss man sich, für die vorliegende Studie die Auswirkungen des Mediengebrauchs der Mütter zu untersuchen.

Um Mutter und Kind für die Videoaufnahmen etwas herauszufordern, gab es in zufälliger Reihenfolge vier Speisen (auf zwei Tellern serviert, jeweils für Mutter und Kind), die sich in Bekanntheit und Süße unterschieden: zwei Gemüse, grüne Bohnen (bekannt) und Artischockenherzen (unbekannt), sowie zwei Desserts, Cupcakes (bekannt) und Halva ${ }^{6}$ (unbekannt). Mutter und Kind saßen zusammen an einem Tisch und erhielten die folgende Instruktion: „Wann immer es Ihnen und [Ihrem Kind] recht ist, werde ich zwei Portionen des ersten Gangs der Mahlzeit bringen. Sie können die Speise probieren oder nicht, und auch [Ihr Kind] kann es probieren oder nicht. Wir machen das mit insgesamt vier unterschiedlichen Gängen. Sie können sie alle probieren und mir sagen, wie Sie es finden. Wenn Sie die Speise nicht versuchen mögen, ist das auch in Ordnung. Okay?" Mutter und Kind bekamen ihre

6 Auch dem Autor war diese „ursprünglich aus Indien, Iran, Pakistan und Zentralasien“ stammende Süßwarenspezialität unbekannt, bei der es sich um einen „Mus von Ölsamen und Zucker oder Honig“ handelt, der durch „Zugabe von Vanille, Kakao, Nüssen, Mandeln oder Pistazien [...] verfeinert bzw. aromatisiert" wird. (https://de.wikipedia.org/w/in dex.php?title=Halva\&oldid=17429321; abgerufen am 1.7.2018)
Speisen und es wurde ihnen jeweils auch gesagt, worum es sich handelt. Dann wurde die Mutter gefragt, ob sie oder ihr Kind das schon einmal gegessen hätten. Und dann wurde noch gesagt: „Versuchen Sie es, wenn Sie mögen, und sagen Sie mir, was Sie darüber denken, wenn ich in ein paar Minuten wiederkomme ${ }^{\text {"7 }}$ (23, S. 239; Übersetzung durch den Autor).

Mutter und Kind wurden dann für jeweils vier Minuten allein gelassen. Sie wussten, dass in dieser Zeit eine Videoaufnahme von ihnen aufgezeichnet wurde, und wurden danach jeweils gefragt, wie sie das Essen fanden. Digitale Medien wurden mit keinem Wort angesprochen. Wie die Analyse der Videoaufnahmen ergab, benutzten zwei Drittel der Mütter (66,7\%) während der Mahlzeit keine digitalen $\mathrm{Ge}$ räte, weitere $10,2 \%$ schauten nur kurz danach oder hatten ihr Gerät auf dem Tisch liegen. Knapp ein Viertel der Mütter $(23,1 \%)$ verwendete während der Mahlzeit ihr mobiles digitales Endgerät mindestens einmal für längere Zeit.

Um genau nachzusehen, was die Mediennutzung der Mütter bei ihnen bewirkt, wurde ihr Verhalten nach einem einheitlichen Standard kodiert, um danach eine multivariate Regressionsanalyse rechnen zu können. Verwendet wurde ein Kodierungsschema, das sich Bob and Tom's Method of Assessing Nutrition - abgekürzt: BATMAN (kein Witz!) - nennt. Es unterscheidet verbale (,probier mal'nen Bissen“) sowie non-verbale (die Mutter gibt dem Kind einen Bissen) Ermunterungen und Akte des Abratens (verbal: „das sieht aber nicht so gut aus"; non-verbal: die Mutter schiebt den Teller etwas weg vom Kind). Aus diesen vier Variablen wurden die fol-

\footnotetext{
7 "Once you and [your child] are comfortable, I will bring 2 servings of a food into the room. You can either choose to try it or not. [Your child] can either choose to try it or not. We will do this with 4 different foods. You are welcome to give them a try and tell me what you think of them. If you really don't want to try them, though, you don't have to. Okay?" The mother and child were given individual servings, a research assistant identified the food for them (eg, "These are artichokes. It is a kind of vegetable"), asked mother if she or the child had ever tried the food before, and said to both: "Give it a try if you'd like, and tell me what you think of it when I come back in a couple of minutes."
} 

genden vier umgeformten Variablen berechnet:

- das Ausmaß der verbalen und

- der non-verbalen Interaktionen (des Ermunterns und Abratens) sowie

- das Ausmaß der (verbalen und nonverbalen) Ermunterung und

- des (verbalen und non-verbalen) Abratens.

Diese Variablen wiederum wurden über alle vier Speisen zusammen sowie für jede Speise einzeln berechnet (23, S.240).

Bei den Müttern mit Handygebrauch während des Essens wurden signifikant weniger verbale Interaktionen $(11,1$ versus 14,1; $\mathrm{p}<0,05)$ mit ihren Kindern beobachtet, insbesondere während des Essens von Halva, der unbekanntesten Speise (2,3 versus 3,7; $p=0,03)$. Diese Mütter ermunterten ihr Kind auch seltener zum Essen (8,8 versus 12,$3 ; p=0,03)$, und wieder gab es insbesondere beim Essen von Halva weniger Ermunterungen (1,9 versus 3,5; $\mathrm{p}=$ 0,02). Auf die Anzahl der Akte des Abratens von Speisen durch die Mutter hatte ihr Medienverhalten keinen Einfluss.

Eine multivariate Regressionsanalyse der Essverhaltensweisen, die weitere in solchen Studien übliche Kontrollvariablen (Alter, Bildung, sozioökonomischer Status, ethnische Zugehörigkeit) berücksichtigte, zeigte zudem Folgendes: Benutzte die Mutter während der Mahlzeit ein Mobiltelefon oder einen Tablet-Computer, kam es zu $20 \%$ weniger verbalen und zu $39 \%$ weniger non-verbalen Interaktionen zwischen Mutter und Kind. Dieser Zusammenhang war für unbekanntere Speisen stärker, mit 26\% weniger verbalen und zu $48 \%$ weniger nonverbalen Interaktionen als für bekanntere Speisen. Am deutlichsten war der Effekt bei der unbekanntesten Speise, Halva, mit $33 \%$ weniger verbalen und zu $58 \%$ weniger non-verbalen Interaktionen.

Eine multivariate Regressionsanalyse der Essverhaltensweisen, die weitere in solchen Studien übliche Kontrollvariablen (Alter, Bildung, sozioökonomischer Status, ethnische Zugehörigkeit) berücksichtigte, zeigte zudem Folgendes: Benutzte die Mutter während der Mahlzeit ein Mobiltelefon oder einen Tablet-Computer, kam es zu $20 \%$ weniger verbalen und zu $39 \%$ weniger non-verbalen Interaktionen zwischen Mutter und Kind. Dieser Zusammenhang war für unbekanntere Speisen stärker, mit 26\% weniger verbalen und zu $48 \%$ weniger nonverbalen Interaktionen als für bekanntere Speisen. Am deutlichsten war der Effekt bei der unbekanntesten Speise, Halva, mit $33 \%$ weniger verbalen und zu $58 \%$ weniger non-verbalen Interaktionen $(\triangleright$ Abb. 1).

Die Nutzung eines digitalen Endgeräts durch die Mutter während der Mahlzeit führte zu einer Reduktion der Anzahl an Ermunterungen zum Essen.

Was die Ermunterungen zum Essen anbelangt, ergab sich ein ähnliches Bild ( $\triangle \mathrm{Abb}$. 1): Die Nutzung eines digitalen Endgeräts durch die Mutter während der Mahlzeit führte zu einer Reduktion der Anzahl an Ermunterungen um 28\% (alle Speisen),

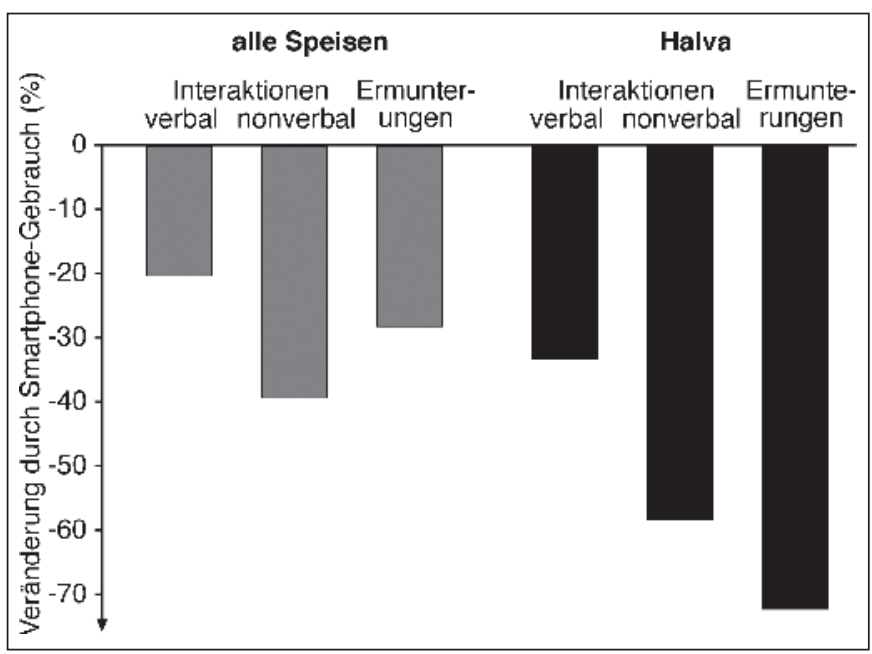

Abb. 1

Prozentuale Verminderungen der Interaktionen zwischen Mutter und Kind und der Ermunterungen des Kindes durch die Mutter bei allen Speisen und bei der am wenigsten bekannten Speise Halva (nach Daten aus 23 , S. 240)
35\% (unbekannteren Speisen, d. h. Halva und Artischockenherzen) bzw. 72\% (Halva).

Der Befund, dass die Nutzung mobiler digitaler Geräte das mütterliche Engagement (sowohl die Anzahl der Interaktionen als auch die Zahl der Ermunterungen) vor allem dann reduziert, wenn es um für das Kind neue Erlebnisse geht, ist den Autoren zufolge von besonderer Bedeutung. Neue Erfahrungen bewirken Lernen, und hierbei braucht das Kind Unterstützung, ganz gleich, ob es sich um neue Speisen oder neue andere Erfahrungen handelt. Wenn also gerade neue Erfahrungen und damit Lernprozesse durch die Smartphone-Nutzung der Mutter beeinträchtigt werden, muss dies negative Konsequenzen für die kindliche Entwicklung haben. Bedenkt man nun noch, dass weltweit mehr als 5 Milliarden Smartphones in Gebrauch sind und dass Mahlzeiten häufige, wesentliche Anlässe für familiäres Miteinander und damit auch für häusliche Lernprozesse sind, so kann man die Bedeutung der hier vorgestellten Befunde kaum überschätzen.

In einer weiteren Studie wurde an einer Teilgruppe von 195 Mutter-Kind-Dyaden (der gerade beschriebenen 225) noch der Frage nachgegangen, wovon es abhängt, ob die Mutter während einer Mahlzeit zum Smartphone griff oder nicht. Die Mütter wurden hierzu strukturiert nach ihrer Einstellung zu ihrem Kind befragt. Es zeigte sich, dass die Wahrnehmung des Kindes als „schwierig“ mit der Verwendung von Smartphones bei den Mahlzeiten assoziiert war: „Specifically, we found positive associations between active mobile device use during family meals with the perception of the child as difficult" (26, S. 314).

Weitere Studien zur Smartphone-Nutzung von Eltern aus der gleichen Arbeitsgruppe ergaben das häufige Auftreten von "Aufregung und Erschöpfung" (24) im Rahmen der folgenden drei Herausforderungen:

- Mit dem Smartphone versuchen Eltern, Arbeit und Kinder möglichst gleichzeitig im Griff zu haben;

- das Smartphone verursacht emotionale Spannungen (die man mit seiner Benutzung abzubauen versucht) sowie Spannungen im Umgang mit Kindern, die selber ihr Smartphone zu oft nutzen. 
Eltern möchten sie einerseits ermahnen, wollen aber andererseits auch ihre Ruhe und den Frieden mit den Kindern.

Eine kürzlich im Fachblatt Pediatric Research publizierte Längsschnittstudie an 183 Elternpaaren mit Kindern unter fünf Jahren konnte das komplexe Ursache-Wirkungs-Gefüge der Verhaltensweisen von Eltern und ihren Kindern noch besser beschreiben (14). Im Zeitraum von 2014 bis 2016 wurden Eltern an vier Zeitpunkten (Baseline sowie Wiederholungsmessungen nach einem Monat, nach drei und nach sechs Monaten) mittels standardisierter Instrumente befragt. Zum einen wurden Störungen durch digitale Medien mittels der Technology Device Interference Scale (TDIS) erhoben. Die Frage „Wie oft unterbrechen die folgenden Geräte ein Gespräch oder eine Aktivität mit Ihrem Kind an einem normalen Tag" wird hierbei für 6 Gerätetypen (Mobiltelefon/Smartphone, Fernseher, Computer, Tablet, iPod und Spielekonsole) einzeln auf einer 7-stufigen Skala von 0 (gar nicht) bis 6 (über $20 \mathrm{Mal}$ ) beantwortet. Kindliche Verhaltensprobleme wurden getrennt nach externalisierenden (Hyperaktivität, geringe Frustrationstoleranz, Aggressivität, Wutanfälle) und internalisierenden Problemen (Weinerlichkeit, Schmollen, Verletztheit) sowohl durch den Vater als auch durch die Mutter nach der Child Behavioral Checklist (CBCL) eingeschätzt, wobei jeweils auf die vergangenen 2 Monate Bezug genommen werden sollte. Zudem wurde der elterliche Stress mit 27 Items aus dem Parenting Stress Index (PSI), das elterliche Miteinander mit der Coparenting Relationship Scale $(C R S)^{8}$ und depressive Symptome bei Mutter und Vater durch die Epidemiologic Studies Depression Scale (CES-D), bestehend aus 20 Items, erfragt sowie die für derartige Studien üblichen Kovariablen (Eltern: Alter, Bildungsgrad, Familienstand, ethnische Herkunft, Einkommen; Kind: Alter, Geschlecht und tägliche Mediennutzungszeit, getrennt nach 8 Gerätetypen).

Es zeigte sich insgesamt, dass es an fast allen Tagen zu Unterbrechungen des Aus-

8 Diese Skala besteht aus 35 Items, die von „Wenn ich am Ende bin, gibt mir mein Partner die extra Unterstützung die ich brauche“ bis „Mein Partner unterminiert meine Elternschaft" reichen (6). tauschs zwischen Eltern und Kind durch digitale Endgeräte kam: Über alle vier Messzeitpunkte hinweg gaben Väter die Anzahl der Geräte, die täglich stören, im Mittel mit 1,43 und Mütter mit 1,65 an. Über keinerlei tägliche Störungen berichteten nur 4,9\% der Mütter und 9,6\% der Väter, wohingegen $55,5 \%$ der Mütter und $43,0 \%$ der Väter über Störungen durch 2 oder mehr Geräte täglich berichteten - unabhängig von Einkommen, Alter, Bildungsgrad oder ethnischer Herkunft der Eltern. Sowohl bei den Müttern als auch bei den Vätern zeigte sich, dass diese Unterbrechungen mit signifikant häufigeren Verhaltensauffälligkeiten beim Kind (sowohl Internalisierung als auch Externalisierung) und mit signifikant mehr Stress bei den Eltern einhergingen. Die Unterbrechungen verminderten auch die Qualität des elterlichen Miteinanders (Coparenting) signifikant und führten bei den Müttern zu signifikant mehr Depressivität.

Durch die wiederholten Befragungen war es möglich, Auswirkungen der gemessenen Variablen zu einem früheren Zeitpunkt auf diese Variablen zu späteren Zeitpunkten statistisch zu untersuchen. Hierbei zeigte sich Folgendes:

$\mathrm{Zu}$ Beginn der Studie (Baseline) erfasste technikbedingte Unterbrechungen seitens der Eltern gingen mit Verhaltensproblemen der Kinder zu späteren Zeitpunkten einher. Bei externalisierendem Problemverhalten war dies zu allen Zeitpunkten (d. h. nach einem, drei und sechs Monaten) der Fall. Beim internalisierenden Problemverhalten sagte nur dagegen die Messung der Unterbrechungen zum Zeitpunkt „3 Monate" das Verhalten zum Zeitpunkt „6 Monate" vorher.

Verhaltensprobleme beim Kind zu Beginn der Studie (Baseline) führten an allen späteren Messzeitpunkten bei den Eltern zu erhöhtem Stress. Wieder war dies vor allem bei externalisierendem Problemverhalten der Kinder (und weniger bei internalisierendem) zu verzeichnen.

Mehr elterlicher Stress führt dazu, dass sie zu späteren Zeitpunkten mehr Unterbrechungen vornehmen, d. h. im Klartext: Wenn die Eltern Stress mit ihren Kindern haben, wenden sie sich eher von den Kindern ab und dem Smartphone zu.
Wenn die Eltern Stress mit ihren Kindern haben, wenden sie sich eher von den Kindern ab und dem Smartphone zu.

Auch wenn das internalisierende Verhalten einen insgesamt geringeren Effekt hatte, zeigten sich bei Betrachtung nur eines der Symptome internalisierender Verhaltensauffälligkeiten - dem Rückzug des Kindes - deutliche Auswirkungen: Mehr Unterbrechungen zu Beginn der Studie (Baseline) bewirkten später mehr Rückzug des Kindes, der wiederum den Eltern mehr Stress machte, was wiederum danach $\mathrm{zu}$ (noch) mehr Unterbrechungen durch die Eltern führte.

Insgesamt zeigt die Studie, dass digitale Medien die Eltern-Kind-Beziehung in mehrfacher Weise deutlich beeinträchtigen: Sie führen beim Kind zu Verhaltensproblemen, was wiederum bei den Eltern eine noch größere Neigung zu solchen Unterbrechungen mittels digitaler Medien bewirkt. Der oben bereits angesprochene Teufelskreis aus elterlicher SmartphoneNutzung während ihres Kontakts zu den Kindern und kindlichen Verhaltensproblemen wurde damit empirisch im Längsschnitt erstmals direkt bestätigt.

Die Studie zeigt, dass digitale Medien die Eltern-Kind-Beziehung in mehrfacher Weise deutlich beeinträchtigen.

Die Interpretation dieser Ergebnisse durch die Autoren sei dem Leser nicht vorenthalten: „Wir denken, dass bestimmte Eigenschaften der digitalen Technologie, einschließlich ihres verführerischen Designs (eine lange Nutzungszeit hat eine belohnende Wirkung), besonders solche Eltern ansprechen, die entweder ohnehin Schwierigkeiten mit der eigenen Selbstregulation haben oder die mit ihrem Familienleben frustriert sind. Dies wiederum führt $\mathrm{zu}$ noch mehr Unterbrechungen durch digitale Technologie als ohnehin schon geschehen" $(15$, S. 7).

Weitere Studien bestätigen und erweitern diese Ergebnisse: Die Nutzung von digitalen Medien führt bei den Müttern zu mehr Depressivität (13) und bei beiden Eltern zu mehr Streit, mehr Problemen bei 
der gegenseitigen Unterstützung und zu einer geringeren Zufriedenheit mit der Beziehung und vor allem des elterlichen Miteinanders (16). Eine Studie an 183 Paaren mit Kindern zeigte, dass der Mediengebrauch der Eltern zu mehr Verhaltensproblemen bei ihren Kindern führt (15).

Damit werden die schlimmsten Befürchtungen bestätigt, vor allem dann, wenn man den Medienkonsum durch die Kinder und Jugendlichen selbst mit einbezieht. Dieser hat negative Auswirkungen auf die Entwicklung der Kinder, wie jüngst auch durch zwei deutsche Studien belegt wurde. Die BLIKK-Studie (das Akronym steht für Bewältigung Lernverhalten Intelligenz Kompetenz Kommunikation) wurde in 79 deutschen Kinder- und Jugendarztpraxen an 5573 Kindern und Jugendlichen (und deren Eltern) durchgeführt und führte die Ergebnisse aus den jeweiligen U- und J-Untersuchungen mit denen von Befragungen zusammen $(1,5)$. Ihre wesentlichen Ergebnisse sind:

- $70 \%$ der Kinder im Kita-Alter benutzen das Smartphone ihrer Eltern mehr als eine halbe Stunde täglich.

- Es gibt einen Zusammenhang zwischen einer intensiven Mediennutzung und Entwicklungsstörungen der Kinder. Diese sind altersabhängig und können wie folgt beschrieben werden.

- Mütter, die beim Stillen ihrer Säuglinge (Alter: 4 Wochen bis 12 Monate; U3 bis U6; $\mathrm{n}=1828$ ) auf das Smartphone schauen, haben mehr Schwierigkeiten beim Füttern und unruhiger schlafende Kinder.

- Die Smartphone-Nutzung der 2- bis 5-jährigen Kinder (U7 bis U9; $\mathrm{n}=2060$ ) geht mit Konzentrationsstörungen und Sprachentwicklungsstörungen einher.

- Bei 8- bis 14-Jährigen (U10 bis J1; n = 1685) bewirkt die Smartphone-Nutzung Konzentrationsstörungen und Übergewicht.

- Bei den Jugendlichen (13-14 Jahren; n = 535) kommt es zudem zu Problemen, die eigene Smartphone- und Internetnutzung selbstbestimmt zu kontrollieren.

Eine im Mai 2018 publizierte einjährige Längsschnittstudie an insgesamt 537 Vorschulkindern im Alter von zwei bis sechs
Jahren aus der Leipziger Kinderstudienambulanz LIFE Child hatte ähnliche beunruhigende Ergebnisse (21):

- Die Nutzung von Smartphones durch Vorschulkinder stieg zwischen 2011 und 2016 deutlich an.

- Smartphones werden von Kindern aus Familien mit niedrigem Einkommen in höherem Ausmaß benutzt.

- Je mehr 2- bis 6-Jährige mit Computer und Internet zum ersten Zeitpunkt der Datenerhebung beschäftigt waren, desto eher haben sie ein Jahr später Schwierigkeiten im Umgang mit anderen Kindern und umso weniger Freunde haben sie im Vergleich zu Kindern ohne digitale Medien.

- Kinder, die viel Zeit mit einem Smartphone verbringen, leiden ein Jahr später häufiger unter Hyperaktivität und Schlafstörungen.

- Auch haben sie mehr Schulschwierigkeiten, insbesondere in Mathematik.

- Kinder, die zum ersten Messzeitpunkt Schwierigkeiten im Umgang mit anderen Kindern hatten, verbrachten ein Jahr später mehr Zeit mit Computer, Internet und Smartphone.

Für die Nutzung von Medien durch Kinder ergibt sich damit der gleiche Teufelskreis wie bei den Eltern: Mehr Schwierigkeiten im sozialen Bereich bewirken mehr Medienkonsum und dieser wiederum führt zu mehr Schwierigkeiten im sozialen Bereich!

Für die Nutzung von Medien durch Kinder ergibt sich der gleiche Teufelskreis wie bei den Eltern.

Diese Ergebnisse beider Studien zeigen deutlich, dass auch in Deutschland Smartphones Kindern schaden. Man kann sich also keineswegs hierzulande damit „herausreden“, dass in den USA der Medienkonsum ja viel ausgeprägter sei, wir das Ganze „moderater“ handhaben würden und daher nichts zu befürchten hätten.

Glücklicherweise gibt es schon seit längerer Zeit wissenschaftliche Erkenntnisse dazu, was ein Kind $\mathrm{zu}$ seiner gesunden Entwicklung braucht und was nicht (9). Es braucht viel unmittelbare Begegnungen mit stabil vorhandenen Erwachsenen (wenn möglich und vor allem: Mutter und Vater) und mit anderen Kindern. Es braucht Millionen von miteinander gesprochenen Wörtern (29), und Zehntausende kleiner „Projekte“, vom Singen eines Liedes, Malen eines Bildes, Fußballspielen, Bäumeklettern und vor allem dem Miteinander-Spielen, oft in bestimmen Rollen („Mutter und Vater“, „Mutter und Kind“, „Räuber und Gendarm“, „Cowboy und Indianer“, „Lehrer und Schüler“, „Verkäufer und Käufer"). Dabei lernt man nicht nur singen, malen, Fußball spielen, klettern, sondern lernt das Erkennen von Emotionen und Intentionen eines anderen Kindes aus verbalen und non-verbalen Anzeichen (32), Empathie und damit soziales Verhalten (27). Man lernt vor allem auch eines: Sein Ding zu machen, d. h. eine Idee (Lied, Bild, Aktion, Geschichte etc.) in die Realität umzusetzen. Früher sprach man von „Willenskraft", heute von mentaler Stärke oder - in der Wissenschaft - von „exekutiven Funktionen“, von denen bekannt ist, dass ihre Entwicklung uns zu gesünderen, glücklicheren Menschen macht, die zudem im Durchschnitt noch genau deswegen ein höheres Einkommen haben und länger leben (19).

Seit es das Fernsehen gibt, wissen wir, dass Erwachsene weniger sprechen (die Anzahl der Wörter nimmt messbar ab; 3), ihren Kindern weniger Aufmerksamkeit schenken (11) und die Qualität ihres Umgangs mit den Kindern sinkt (17), wenn der Fernseher läuft. Mit dem Aufkommen der Smartphones hat sich diese Situation nun verschärft, denn wir haben sie immer und überall dabei und sie lenken uns von unseren Aufgaben (33) und von unseren Mitmenschen (18) ab, selbst wenn sie nur herumliegen und nicht verwendet werden (30). Die Dosis macht das Gift! Wenn dieser bekannte Satz auch im Hinblick auf die Auswirkungen von Bildschirmen auf die Entwicklung von Kindern gilt, dann „überdosieren" wir Bildschirme mit unseren Smartphones in völlig aberwitzigem und unverantwortlichem Ausmaß.

\section{Die Dosis macht das Gift!}

Wird mit Kindern wenig gesprochen, werden sie vernachlässigt und haben sie wenig Gelegenheit zum Spielen mit anderen Kindern, dann entwickeln sie ihre Potenziale nicht. Medien schaden ihrer Entwicklung, 
denn von Medien lernt kein Kind die Muttersprache, Medien lenken ab und führen dazu, dass Kinder reagieren, wo sie früher agierten (d. h. ihre eigenen Ideen in die Tat umsetzten). Wenn Kinder selbst zu früh zu viel Zeit mit Medien verbringen (was bei den heute üblichen 6 bis 9 Stunden täglich definitiv der Fall ist), schadet dies nachweislich ihrer Entwicklung.

Hinzu kommen die hier diskutierten Auswirkungen der Mediennutzung ihrer Eltern, die mit (in den USA) durchschnittlich täglich 9 Stunden und 22 Minuten extreme Werte erreicht hat. Weil diese Nutzung vor allem in die Freizeit fällt, kommt es dadurch - wie in dieser Arbeit dargestellt - zu einer weiteren, zusätzlichen Beeinträchtigung der Kinder. Digitale Medien schaden der Entwicklung von Kindern nicht nur, wenn diese sie selbst nutzen, sondern auch, wenn ihre Eltern sie nutzen, während sie ihren Aufgaben als Eltern nachkommen. Das sollten alle Eltern wissen.

\section{Literatur}

1. BLIKK Medien Studie 2018 Abschlussbericht BLIKK-Medien: Kinder und Jugendliche im Umgang mit elektronischen Medien (https://www. drogenbeauftragte.de/fileadmin/Dateien/5_Publi kationen/Praevention/Berichte/Abschlussbericht_BLIKK_Medien.pdf; abgerufen am 2.7.2018).

2. Brand M, Young KS, Laier C. Prefrontal control and Internet addiction: a theoretical model and review of neuropsychological and neuroimaging findings. Front Hum Neurosci 2014; 8: 375.

3. Christakis DA, Gilkerson J, Richards JA, Zimmerman FJ, Garrison MM, Xu D, Gray S, Yapanel U. Audible television and decreased adult words, infant vocalizations, and conversational turns: a population-based study. Arch Pediatr Adolesc Med 2009; 163: 554-558.

4. Davidov M, Grusec JE. Untangling the links of parental responsiveness to distress and warmth to child outcomes. Child Dev 2006; 77: 44-58.

5. Drogenbeauftragte der Bundesregierung $2017 \mathrm{Ge}$ meinsame Pressemitteilung. Ergebnisse der BLIKK Studie 2017 vorgestellt: Übermäßiger Medienkonsum gefährdet Gesundheit von Kindern und Jugendlichen. Berlin 29.5.2017.

6. Feinberg ME, Brown LD, Kan ML. A multi-domain self-report measure of coparenting. Parenting 2012; 12: 1-21.

7. Harris T. How Technology is Hijacking Your Mind - from a Magician and Google Design Ethicist 2016 (https://journal.thriveglobal.com/how-tech nology-hijacks-peoples-minds-from-a-magician- and-google-s-design-ethicist-56d62ef5edf3; abgerufen am 24.6.2018).

8. Hinkley T, Verbestel V, Ahrens W, Lissner L, Molnár D, Moreno LA, Pigeot I, Pohlabeln H, Reisch LA, Russo P, Veidebaum T, PhD; Tornaritis M, Williams G, De Henauw S, De Bourdeaudhuij I; für das IDEFICS Consortium. Early childhood electronic media use as a predictor of poorer wellbeing: a prospective cohort study. JAMA Pediatr 2014; 168: 485-492.

9. Johnson SB, Riley AW, Granger DA, Riis J. The science of early life toxic stress for pediatric practice and advocacy. Pediatrics 2013; 131: 319-327.

10. Kellershohn J, Walley K, West B, Vriesekoop. Young consumers in fast food restaurants: technology, toys and family time. Young Consumers 2018; 19: 105-118.

11. Kirkorian HL, Pempek TA, Murphy LA, Schmidt ME, Anderson DR. The impact of background television on parent-child interaction. Child Dev 2009; 80: 1350-1359.

12. Lauricella AR, Cingel DP, Beaudoin-Ryan L, Robb MB, Saphir M, Wartella EA. 2017 The Common Sense census: Plugged-in parents of tweens and teens. Common Sense Media, San Francisco, CA (http://cmhd.northwestern.edu/wp-content/ uploads/2017/04/common-sense-parent-census_whitepaper_new-for-web.pdf; abgerufen am 28.6.2018).

13. McDaniel BT, Coyne SM. „Technoference“: The interference of technology in couple relationships and implications for women's personal and relational well-being. Psychol Pop Media Cult 2016; 5: 85 .

14. McDaniel BT, Radesky JS. Technoference: longitudinal associations between parent technology use, parenting stress, and child behavior problems. Pediatric Research 2018; June 13; https://doi. org/10.1038/s41390-018-0052-6).

15. McDaniel BT, Radesky, JS. Technoference: parent distraction with technology and associations with child behavior problems. Child Dev 2018; 89: 100-109.

16. McDaniel BT, Galovan AM, Cravens JD, Drouin M. "Technoference" and implications for mothers' and fathers' couple and coparenting relationship quality. Comput Human Behav 2018; 80: 303-313.

17. Mendelsohn AL, Berkule SB, Tomopoulos S, et al Infant television and video exposure associated with limited parent-child verbal interactions in low socioeconomic status households. Arch Pediatr Adolesc Med 2008; 162: 411-417.

18. Misra S, Cheng L, Genevie J, Yuan M. The iPhone effect: the quality of in-person social interactions in the presence of mobile devices. Environ Behav 2016; 48: 275-298.

19. Moffitt TE, Arsenault L, Belsky D, Dickson N, Hancox RJ, Harrington $H$, Houts R, Poulton R, Roberts BW, Ross S, Sears MR, Thomson WM, Caspi A. A gradient of childhood self-control predicts health, wealth, and public safety. PNAS 2011; 108: 2693-2698.

20. Oduor E, Neustaedter C, Odom W, Tang A, Moallem $\mathrm{N}$, Tory $\mathrm{M}$ et al. (eds.) The frustrations and benefits of mobile device usage in the home when co-present with family members. In Proc. of the
2016 ACM Conference on Designing Interactive Systems. ACM, New York, NY 2016.

21. Poulain T, Vogel M, Neef M, Abicht F, Hilbert A, Genuneit J, Körner A, Kiess W. Reciprocal associations between electronic media use and behavioral difficulties in preschoolers. International Journal of Environmental Research and Public Health 2018; $15: 814$

22. Przybylski AK, Weinstein N. Can you connect with me now? How the presence of mobile communication technology influences face-to-face conversation quality. J Soc Pers Relat 2013; 30: 237-246.

23. Radesky JS, Miller AL, Rosenblum KL, Appugliese D, Kaciroti N, Lumeng JC. Maternal mobile device use during a structured parent-child interaction task. Acad Pediatr 2015; 15: 238-244

24. Radesky JS, Kistin CJ, Eisenberg S, Gross J, Block G, Zuckerman B, Silverstein M. Parent perspectives on their mobile technology use: the excitement and exhaustion of parenting while connected. J Dev Behav Pediatr 2016; 37: 694-701.

25. Radesky JS, Kistin CJ, Zuckerman B, Nitzberg K, Gross J, Kaplan-Sanoff M, Augustyn M, Silverstein M. Patterns of mobile device use by caregivers and children during meals in fast food restaurants. Pediatrics 2014; 133: e843-e849.

26. Radesky JS, Leung C, Appugliese D, Miller AL, Lumeng JC, Rosenblum KL. Maternal mental representations of the child and mobile phone use during parent-child mealtimes. J Dev Behav Pediatr 2018; 39: 310-317.

27. Richards R, McGee R, Williams SM, Welch D, Hancox RJ. Adolescent screen time and attachment to peers and parents. Arch Pediatr Adolesc Med 2010; 164: 258-262.

28. Spitzer M. Familienabendessen. Nervenheilkunde 2014; 33: 759-760.

29. Spitzer M. Am Anfang war das Wort. Nervenheilkunde 2015; 34: 466-468.

30. Spitzer M. Die Smartphone-Denkstörung. Nervenheilkunde 2017; 36: 587-590.

31. Steiner-Adair C, Barker TH. The big disconnect: Protecting childhood and family relationships in the digital age. Harper Business 2013.

32. Uhls YT, Michikyan M, Morris J, Garcia D, Small GW, Zgourou E, Greenfield PM. Five days at outdoor education camp without screens improves preteen skills with nonverbal emotion cues. Comput Human Behav 2014; 39: 387-392.

33. Ward AF, Duke K, Gneezy A, Bos MW. Brain Drain: The mere presence of one's own smartphone reduces available cognitive capacity. Journal of the Association for Consumer Research (JACR) 2017; 2: 140-154.

34. Wartella E, Rideout V, Lauricella A. Parenting in the Age of Digital Technology 2014 (http://cmhd. northwestern.edu/wp-content/uploads/ 2015/06/ParentingAgeDigital Technology.REVISED.FINAL_.2014.pdf. 2014; abgerufen am 28.6.2018).

35. Spitzer M. Smartphone und Depression: Ursache oder Therapie? Nervenheilkunde 2018; 37: 7-15.

36. Spitzer M. www (WeltWeite Werbung1) und die Folgen. Radikalisierung, Spionage, Vertrauensund Wahrheitsverlust Nervenheilkunde 2018; 37: 303-311. 